\title{
Primary Vulval Rhabdoid Tumor in an Adult: A Case Report, Immunohistochemical Profile and Literature Review
}

\author{
Maria A. Arafah ${ }^{1,2}$ and Muna I. Aljuboury ${ }^{3}$ \\ ${ }^{1}$ Department of Pathology, College of Medicine, King Saud University, Riyadh 11421, Saudi Arabia \\ ${ }^{2}$ Department of Pathology, College of Medicine and King Khalid University Hospital, P.O. Box 2925, Riyadh 11461, Saudi Arabia \\ ${ }^{3}$ Department of Pathology, Riyadh Armed Forces Hospital, Riyadh 11159, Saudi Arabia
}

Correspondence should be addressed to Maria A. Arafah, marafah83@gmail.com

Received 31 May 2011; Accepted 8 August 2011

Academic Editor: Mark E. Shaffrey

Copyright (C) 2011 M. A. Arafah and M. I. Aljuboury. This is an open access article distributed under the Creative Commons Attribution License, which permits unrestricted use, distribution, and reproduction in any medium, provided the original work is properly cited.

We report a rare case of primary vulval rhabdoid tumor in an adult. The diagnosis was confirmed using the recently emerging INI1/BAF47 immunostain. We also demonstrate the expression of ER and PR hormonal receptors by the tumor cells.

\section{Introduction}

Extrarenal malignant rhabdoid tumors (EMRTs) are rare and highly aggressive neoplasms. Although they are known to occur during infancy and childhood, their occurrence in adults is reported. EMRTs were described in various anatomical sites, and the use of INI1 immunostain has recently emerged as an important adjunct for identifying these tumors. An extensive online search of the literature revealed very few reported cases of EMRT of the vulva [110]. We hereby report another case and the first in which the INI1, ER, and PR immunostains were utilized.

\section{Case Description}

A 25-year-old Saudi female, who is medically free, presented to our emergency department complaining of a mass in the mons pubis. The mass was occasionally tender and had rapidly increased in size in two-week duration. No other complains or masses at other sites were reported. On clinical examination, an ill-defined soft mass was identified in the mons pubis, extending to the left inguinal area with a hyperemic overlying skin. Laboratory tests were within normal limits except for a mild leukocytosis. The clinical impression was of an abscess, and the patient was scheduled for surgery. A local excision of the mass was done, and the specimen was sent to the histopathology department. The patient was discharged on the next day of surgery.

2.1. Gross Findings. Grossly, the specimen consisted of two irregular pieces of soft tissue. Both masses were partially encapsulated and measured $6 \times 5 \times 2 \mathrm{~cm}$ and $6.5 \times 6 \times 2.5 \mathrm{~cm}$, respectively. The outer surface of both masses was irregular. Serial slicing of the masses revealed a heterogeneous cut surface with pale white and dark tan areas. Random sections from both masses were taken for histopathological examination.

2.2. Microscopic Findings. H\&E slides showed a poorly differentiated neoplasm. The tumor consisted exclusively of cells retaining the classical "rhabdoid" morphology (Figure 1). The cells were discohesive with distinct cell borders, and they were arranged in solid sheets. The amount of cytoplasm varied between scant to abundant. The cytoplasm had a prominent eosinophilic quality, and intracytoplasmic glassy eosinophilic inclusions were seen within the majority of the cells. The nuclei were vesicular, eccentric, and highly pleomorphic with prominent nucleoli. Numerous mitoses were seen ( 45 mitoses/10 HPF). Occasional multinucleated tumor giant cells were present. There were neither areas of necrosis nor lymphovascular invasion. 


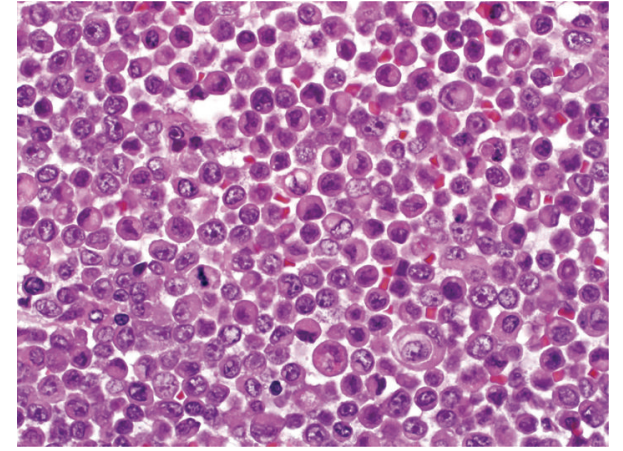

Figure 1: The classical "rhabdoid" morphology of the tumor comprising the cytoplasmic hyaline globules and the eccentric nucleus. Mitotic figures are also seen (H\&E stain, magnification power $\times 400)$.

2.3. Immunohistochemical Findings. An extended panel of antibodies was performed on Ventana using the iVIEW DAB detection kit and showed focal cytoplasmic immunoreactivity to vimentin (mouse monoclonal, 1:60; Dako) with the characteristic globular cytoplasmic configuration indenting the nucleus. The cytoplasm of tumor cells also coexpressed cytokeratin cocktail (mouse monoclonal, AE1/AE3, 1:100; Dako), CK 8 (mouse monoclonal, ready to use; Dako), and CK 19 (mouse monoclonal, 1:40; Dako). The tumor cells, in comparison to the endothelial lining of the capillaries, showed loss of INI1 (mouse monoclonal, BAF 47, ready to use; BD Transduction Laboratories, USA) nuclear staining (Figure 2). Anti-Ki-67 (mouse monoclonal, 1:50; Dako) showed a high proliferation index reaching up to $80 \%$ of tumor cells. Tumor cells have showed nuclear positivity to ER (mouse monoclonal, 1:20; Dako) (weak to moderate in $90 \%$ ) (Figure 3) and have also showed nuclear positivity to PR (mouse monoclonal, 1:100; Dako) (strong in 20\%). All other immunohistochemical (IHC) stains were negative including CD34, EMA, CD99, CD117, CD45, CD30, CD138, bcl-6, bcl-2, CK7, CK20, GCDFP, muscular differentiation markers (Desmin, SMA, Myoglobin), neuroendocrine markers (Synaptophysin, Chromogranin A, CD56), and melanoma markers (S100, HMB-45).

A diagnosis of malignant rhabdoid tumor of the vulva was established. The patient was recalled once the histopathology report was signed out. A computed tomography scan (CT) of the head, chest, abdomen, and pelvis was performed and revealed no other tumor masses. Unfortunately, the patient failed to show for followup.

\section{Discussion}

We report a case of EMRT of the vulva in a 25-yearold female. The age of the patient, the site of the tumor, the clinical presentation, and the microscopic findings in our case were compatible with the 12 previously reported cases (Table 1) [1-10]. The IHC profile varied between cases, and the only universal finding was immunoreactivity to vimentin. Recently, INI1 immunostain has emerged as

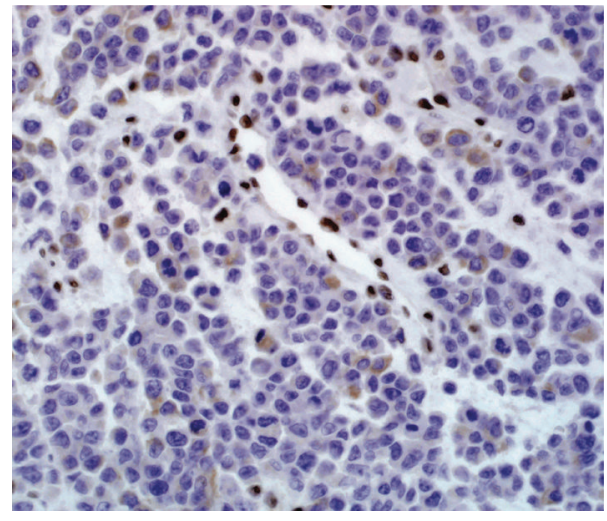

FIGURE 2: INI1/BAF47 immunostain showing loss of nuclear staining in the rhabdoid cells in comparison to the positive nuclei of the endothelial cells (INI1/BAF47 immunohistochemical stain, magnification power $\times 200$ ).

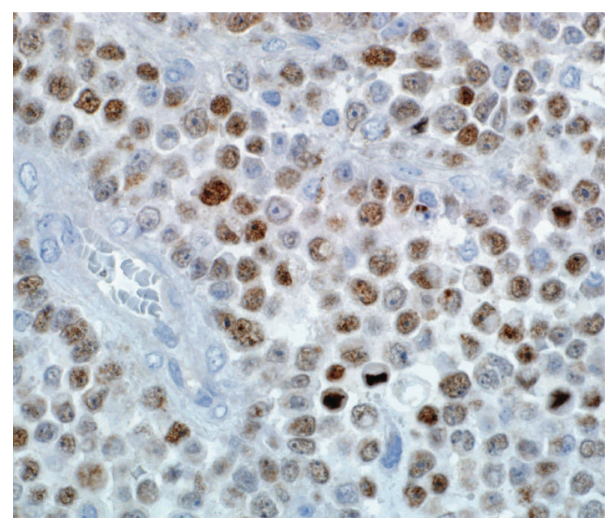

FIGURE 3: Weak-to-moderate ER nuclear expression of the tumor cells (ER immunohistochemical stain, magnification power $\times 400$ ).

an adjunct for identifying rhabdoid tumors and atypical rhabdoid/teratoid tumors (AT/RTs). INI1, encoded by the INI1/hSNF5/SMARCB1/BAF47 locus at 22q11.2, is a member of the SWI/SNF chromatin-remodeling complex, and it is normally expressed in all tissues $[11,12]$. The biallelic inactivation of this gene is characteristic to rhabdoid tumors of the kidney, EMRTs, and AT/RTs $[11,12]$. We have confirmed our diagnosis using the INI1/BAF47 antibody which showed universal loss of nuclear INI1 protein expression in all tumor cells. We have also studied the hormonal status of this tumor and found it to express both ER and PR receptors. This finding is compatible with its primary location in the vulva and has been previously reported in other vulval tumors [13].

Malignant rhabdoid tumor was originally described in the kidney as a variant of Wilms' tumor. In 1982, the first extrarenal malignant rhabdoid tumor was reported, and since then this entity was recognized at various anatomical sites including soft tissues, liver, prostate, thymus, skin, pelvis, heart, and central nervous system [14, 15]. These tumors have a predilection to the pediatric age group though cases in adults were repeatedly recognized. The common microscopic feature is the "rhabdoid" cell of which 
TABLE 1: Characteristics of the previously reported cases of EMRT of the vulva [1-10].

\begin{tabular}{|c|c|c|c|c|}
\hline Author, year & $\begin{array}{l}\text { Age at diagnosis } \\
\text { (years) }\end{array}$ & Site & Primary treatment & $\begin{array}{c}\text { Reported survival } \\
\text { (months) }\end{array}$ \\
\hline \multirow{3}{*}{ Perrone et al., $1989^{\S}[1]$} & 19 & R LMJ & & 38 (alive) \\
\hline & 30 & R LMJ & Surgery & 8 (dead) \\
\hline & 31 & L vulva & & 138 (dead) \\
\hline Matias et al., 1990 [2] & 49 & L LMJ & Surgery + chemotherapy & $9($ dead $)$ \\
\hline Lupi et al., 1996 [3] & 39 & L LMJ & Surgery + chemotherapy + radiation & 4 (dead) \\
\hline Igarashi et al., 1998 [4] & 39 & L LMJ & Surgery + chemotherapy & 8 (alive) \\
\hline Sert et al., 1999 [5] & 44 & R LMJ & Surgery + chemotherapy + radiation & $8($ dead $)$ \\
\hline Brand and Covert $2001[6]$ & 40 & Mons pubis & Surgery + chemotherapy + radiation & 61 (alive) \\
\hline Haidopoulos et al., 2002 $¥$ [7] & - & Clitoris & - & - \\
\hline Tzilinis et al., 2002 [8] & 63 & L LMJ & Surgery + radiation & 30 (alive) \\
\hline Argenta et al., 2007 $€[9]$ & 35 & R vulva & Surgery + radiation & 40 (alive) \\
\hline Narendra et al., 2010 [10] & 50 & R LMJ and mons pubis & Surgery + radiation & 30 (alive) \\
\hline Current case & 25 & $\begin{array}{l}\text { Mons Pubis, extending } \\
\text { to L inguinal area }\end{array}$ & Surgery & Lost followup \\
\hline
\end{tabular}

R: right. L: left. $\S_{3}$ cases were reported. ${ }^{¥}$ No full text is available in PubMed. $€_{\text {The diagnosis given was EMRT/proximal epithelioid sarcoma. }}$

the entire tumor is composed. These tumors usually coexpress vimentin and cytokeratin, and they do not retain the nuclear expression of INI1 protein. The prognosis of ERMT is generally poor, and their course tends to be aggressive. Although surgery is the first line of management, there is no consensus that exists regarding adjuvant therapies. Chemotherapy and radiotherapy did not show to be effective in controlling the recurrent or metastatic disease in all reported cases [1-15].

In summary, vulval EMRTs are rare and aggressive neoplasms and should be considered in the differential diagnosis of any high-grade undifferentiated tumor at this site. Given that IHC is much more widely available than molecular tests, INI1/BAF47 immunostain represents the most useful, up to date ancillary technique to confirm this diagnosis. Although these tumors express ER and PR receptors, their response to antihormonal therapy is a remote possibility [13].

\section{References}

[1] T. Perrone, P. E. Swanson, L. Twiggs, T. M. Ulbright, and L. P. Dehner, "Malignant rhabdoid tumor of the vulva: is distinction from epithelioid sarcoma possible? A pathologic and immunohistochemical study," The American Journal of Surgical Pathology, vol. 13, no. 10, pp. 848-858, 1989.

[2] C. Matias, J. F. M. Nunes, L. F. Vicente, and M. O. Almeida, "Primary malignant rhabdoid tumour of the vulva," Histopathology, vol. 17, no. 6, pp. 576-578, 1990.

[3] G. Lupi, R. Jin, and C. Clemente, "Malignant rhabdoid tumor of the vulva: a case report and review of the literature," Tumori, vol. 82, no. 1, pp. 93-95, 1996.

[4] T. Igarashi, H. Sasano, R. Konno et al., "Malignant rhabdoid tumor of the vulva: case report with cytological, immunohistochemical, ultrastructural and DNA ploidy studies and a review of the literature," Pathology International, vol. 48, no. 11, pp. 887-891, 1998.
[5] M. B. Sert, M. Onsrud, T. Perrone, F. Abbas, and J. L. Currie, "Malignant rhabdoid tumor of the vulva: case report," European Journal of Gynaecological Oncology, vol. 20, no. 4, pp. 258-261, 1999.

[6] A. Brand and A. Covert, "Malignant rhabdoid tumor of the vulva: case report and review of the literature with emphasis on clinical management and outcome," Gynecologic Oncology, vol. 80, no. 1, pp. 99-103, 2001.

[7] D. Haidopoulos, A. Elsheikh, G. Vlahos et al., "Malignant rhabdoid tumor of the clitoris in an elderly patient: report of a case," European Journal of Gynaecological Oncology, vol. 23, no. 5, pp. 447-449, 2002.

[8] A. Tzilinis, L. E. Clarke, C. Affuso, and J. Fessenden, "Successful treatment of malignant rhabdoid tumor of the vulva in an older patient: a case report and review of the literature," Current Surgery, vol. 59, no. 6, pp. 570-571, 2002.

[9] P. A. Argenta, S. Thomas, and J. C. Chura, "Proximal-type epithelioid sarcoma vs. malignant rhabdoid tumor of the vulva: a case report, review of the literature, and an argument for consolidation," Gynecologic Oncology, vol. 107, no. 1, pp. 130-135, 2007.

[10] H. Narendra, S. Ray, L. Rao, and V. Geetha, "Malignant extrarenal rhabdoid tumor of the vulva in an adult," Journal of Cancer Research and Therapeutics, vol. 6, no. 1, pp. 82-85, 2010.

[11] A. C. Hoot, P. Russo, A. R. Judkins, E. J. Perlman, and J. A. Biegel, "Immunohistochemical analysis of hSNF5/INI1 distinguishes renal and extra-renal malignant rhabdoid tumors from other pediatric soft tissue tumors," The American Journal of Surgical Pathology, vol. 28, no. 11, pp. 1485-1491, 2004.

[12] A. Perry, C. E. Fuller, A. R. Judkins, L. P. Dehner, and J. A. Biegel, "INI1 expression is retained in composite rhabdoid tumors, including rhabdoid meningiomas," Modern Pathology, vol. 18, no. 7, pp. 951-958, 2005.

[13] M. R. Nucci and E. Oliva, "Vulvar neoplasia," in Gynecologic Pathology, J. R. Goldblum, Ed., Foundations in Diagnostic Pathology, pp. 33-40, Elsevier Churchill Livingstone, Philadelphia, Pa, USA, 2009. 
[14] F. Gonzalez-Crussi, R. A. Goldschmidt, W. Hsueh, and Y. P. Trujillo, "Infantile sarcoma with intracytoplasmic filamentous inclusions: distinctive tumor of possible histiocytic origin," Cancer, vol. 49, no. 11, pp. 2365-2375, 1982.

[15] R. Kodet, W. A. Newton, N. Sachs et al., "Rhabdoid tumors of soft tissues: a clinicopathologic study of 26 cases enrolled on the intergroup rhabdomyosarcoma study," Human Pathology, vol. 22, no. 7, pp. 674-684, 1991. 


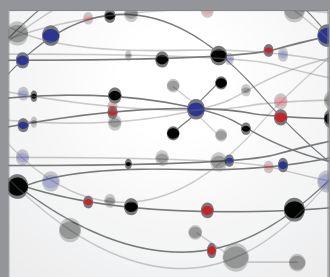

The Scientific World Journal
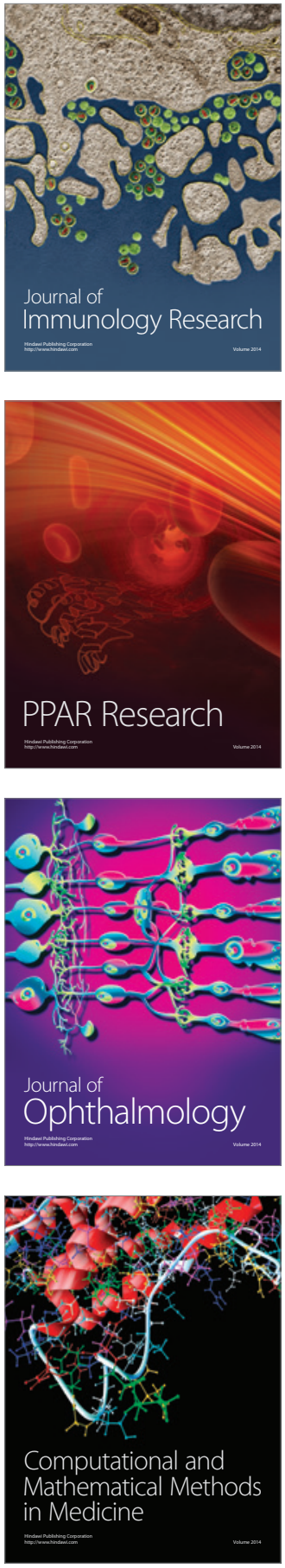

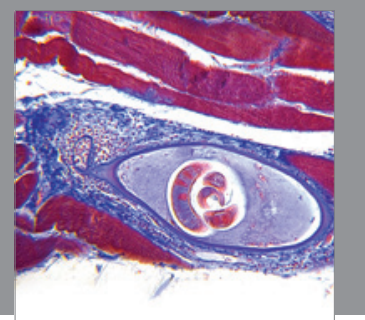

Gastroenterology

Research and Practice
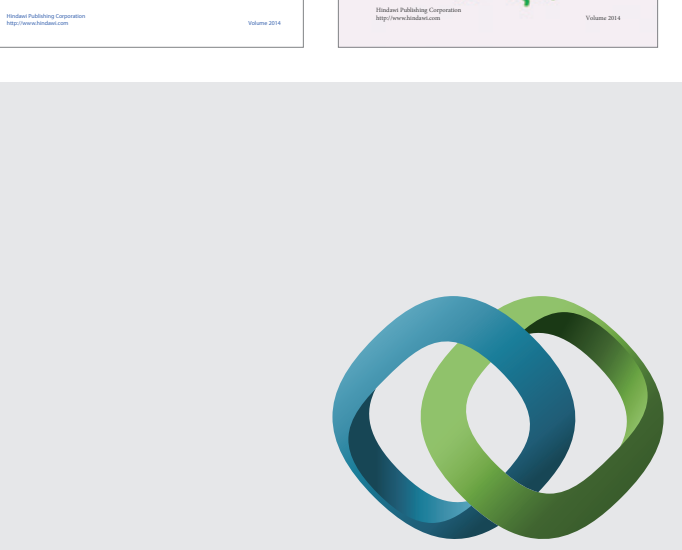

\section{Hindawi}

Submit your manuscripts at

http://www.hindawi.com
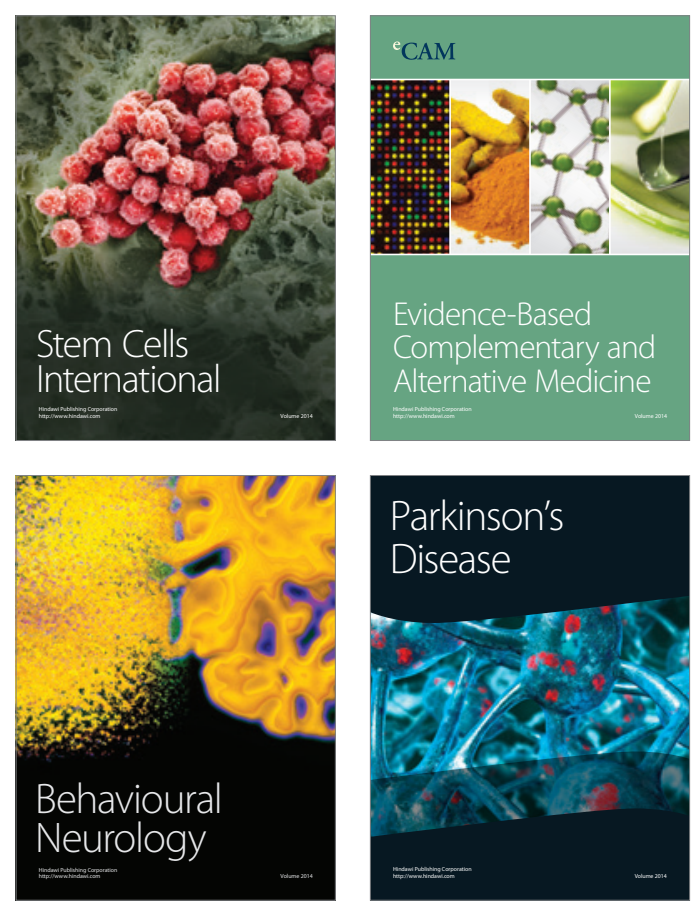

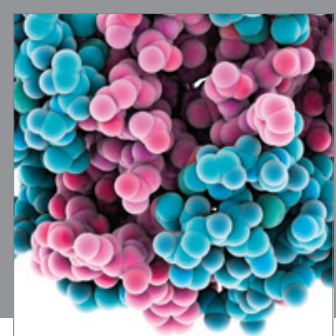

Journal of
Diabetes Research

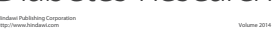

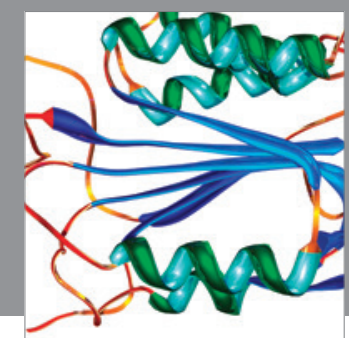

Disease Markers
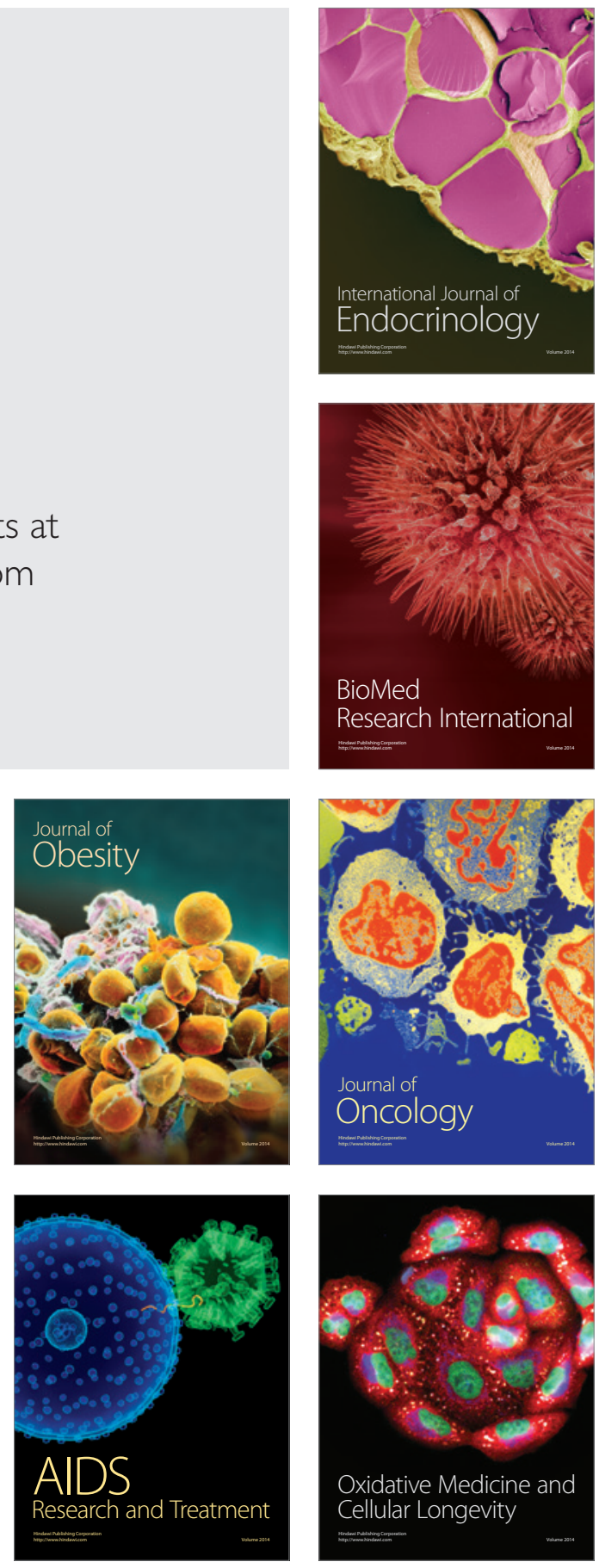